\title{
Intramolecular charge transfer in amino- benzonitriles: Requirements for dual fluorescence
}

\author{
Klaas A. ZACHARIASSE,* Thomas von der HAAR, Axel HEBECKER, \\ Uwe LEINHOS, and Wolfgang KÜHNLE \\ Max-Planck-Institut für biophysikalische Chemie, D-3400 Göttingen, Germany
}

\begin{abstract}
Dual fluorescence and intramolecular charge transfer (ICT) are observed with aminobenzonitriles when two excited state levels $\left(S_{1}\right.$ and $S_{2}(C T)$ in DMABN) have an energy gap sufficiently small for vibronic coupling: a solvent-induced pseudo-Jahn-Teller effect. It is argued that the $\mathrm{N}$-inversion of the amino group acts as a promoting mode. These conclusions are based on a comparison of absorption spectra and photostationary and time-resolved fluorescence data. Dual fluorescence is also observed with MMD, in which the dimethylamino group is twisted towards a perpendicular configuration with respect to the phenyl ring.
\end{abstract}

\section{INTRODUCTION}

The dual fluorescence coupled with intramolecular charge transfer (ICT) in N,N-dimethyl-4-aminobenzonitrile (DMABN) and related molecules has attracted unabated attention since its discovery in 1959 (ref. 1). Among the perhaps surprisingly large variety of mechanisms presented in the literature to explain this phenomenon, the model of "Twisted Internal Charge Transfer" (TICT) pioneered by Grabowski (ref. 2), has played the most prominent role. In this paper, the excited state behaviour of a series of aminobenzonitriles is treated, based on photostationary and time-resolved fluorescence measurements. This behaviour is compared with the differences in the absorption spectra of these compounds, which leads to a discussion of the requirements for ICT.

\section{EXPERIMENTAL SECTION}

The fluorescence spectra were measured by using a quantum-corrected Perkin-Elmer MPF-44E or a Shimadzu 5001 spectrofluorimeter. The picosecond laser single photon counting (SPC) data were obtained employing a laser system described previously (ref. 3), with a Hamamatsu R2809U-07 MCP photomultiplier $(-3300 \mathrm{~V})$ as detector. The instrument response function has a half-width of $35 \mathrm{ps}$ at the excitation wavelength $(296 \mathrm{~nm})$.

\section{RESULTS AND DISCUSSION}

\section{Dual fluorescence}

Dual fluorescence has been observed with $\mathrm{N}, \mathrm{N}$-dialkyl-4-aminobenzonitriles such as DMABN in media with an effective polarity larger than that of the alkane solvents (ref. 1-3). Such a dual fluorescence,

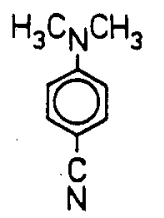

DMABN<smiles>CNc1ccc(C#N)cc1</smiles><smiles>N#Cc1ccc(N)cc1</smiles>

$\mathrm{ABN}$

consisting of emissions from an initially excited aniline-like (LE) and an intramolecular charge transfer (CT) state, could not be detected (ref. 3) with the related compounds N-methyl-4-aminobenzonitrile (MABN) and 4-aminobenzonitrile (ABN), having amino groups with either one or no methyl substituent. The new red-shifted CT emission band is absent even in strongly polar aprotic or protic solvents such as acetonitrile or methanol. 
In addition, the fluorescence decays of MABN and $A B N$ in a variety of solvents, irrespective of their polarity, are single-exponential with lifetimes of several nanoseconds, see Fig. 1 for acetonitrile, indicating that excited state CT reactions do not take place. This behaviour is in clear contrast to that of DMABN, for which rapid reversible ICT in the picosecond time domain is observed (Fig. 1c).

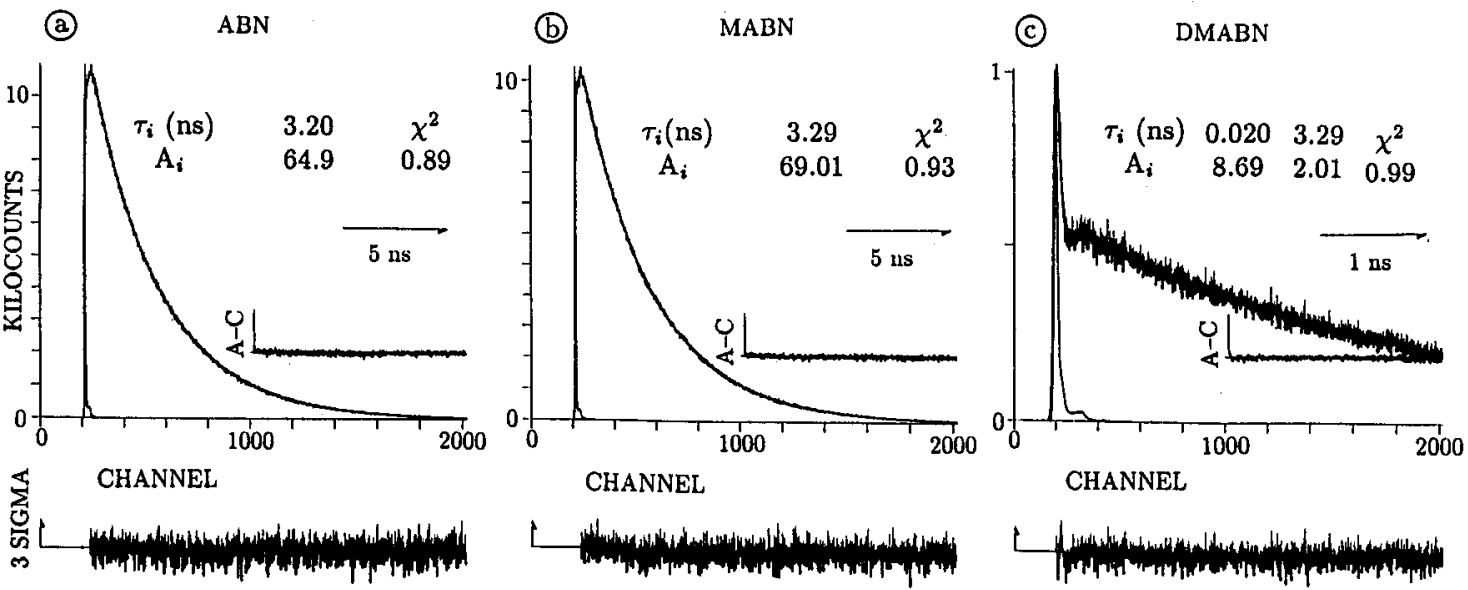

Fig. 1. Fluorescence (LE) decay curves of (a) ABN, (b) MABN and (c) DMABN in acetonitrile at $25^{\circ} \mathrm{C}$.

\section{Comparison of absorption and fluorescence spectra}

Inspection of the absorption spectra of the three 4-aminobenzonitriles in n-heptane $(\epsilon=1.9)$ reveals in the case of $\mathrm{ABN}$, Fig. 2a, the presence of two distinct bands, a relatively weak band $\left(S_{1}\right)$ with
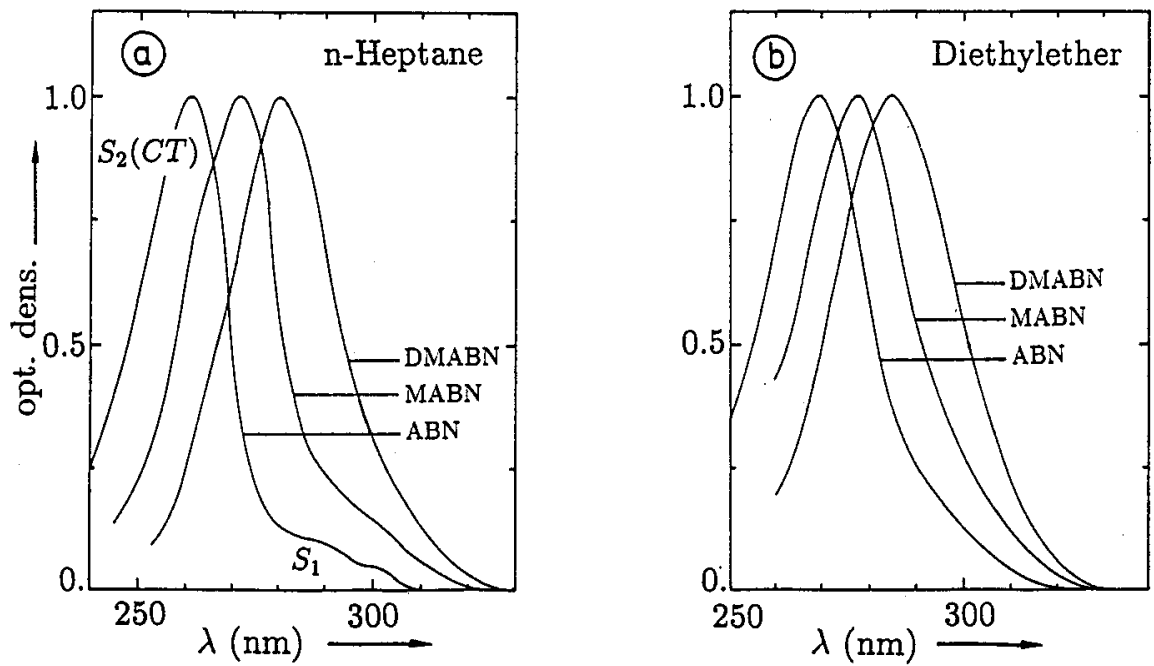

Fig. 2. Absorption spectra of ABN, MABN and DMABN in (a) n-heptane and (b) diethylether at $25^{\circ} \mathrm{C}$.

vibrational structure and a more pronounced structureless absorption band, attributed (ref. 4) to a CT transition $\left(S_{2}(C T)\right)$. For MABN and DMABN the structureless band progressively overlaps with $S_{1}$, although even with DMABN this band can still be seen.

In the somewhat more polar solvent diethylether $(\epsilon=4.3)$, Fig. $2 \mathrm{~b}$, the CT band is more strongly redshifted towards the structured band than in n-heptane, due to the dipolar character of $S_{2}(C T)$. With MABN and ABN the $S_{1}$ band is still clearly visible at the low-energy side of the spectrum, whereas with DMABN $S_{1}$ has disappeared completely under the $S_{2}(C T)$ band. Note in this connection that with DMABN, as the only one of the three 4-aminobenzonitriles, dual tluorescence is observed (ref. 
1a). From this comparison of the absorption and fluorescence spectra of ABN, MABN and DMABN in $\mathrm{n}$-heptane and diethylether, it appears that the onset of dual fluorescence is related to the separation in energy between the lowest excited state $S_{1}$ and $S_{2}(C T)$.

\section{Meta- and ortho- $N, N$-dimethylaminobenzonitriles}

The hypothesis that a small energy gap between $S_{1}$ and $S_{2}(C T)$ is a requirement for the appearance of dual fluorescence is supported by the excited state behaviour of $\mathrm{N}, \mathrm{N}$-dimethyl-3-aminobenzonitrile ( $m$-DMABN) and $\mathrm{N}, \mathrm{N}$-dimethyl-2-aminobenzonitrile (o-DMABN). In the absorption spectra of $\mathrm{m}$ DMABN and o-DMABN the $S_{1}$ and $S_{2}(C T)$ bands are clearly separated (ref. 4), with an energy gap even considerably larger than that found with MABN and ABN. The fluorescence spectra of these two isomers of DMABN in a variety of solvents, from n-pentane to methanol, consist of a single
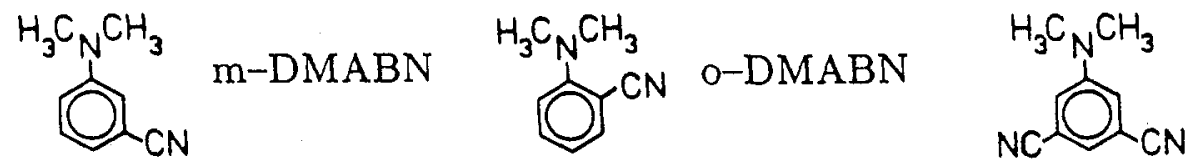

3,5-DCDMA

aniline-like emission, similar to that observed with MABN and ABN. In accordance with this observation, the fluorescence decays of $m-D M A B N$ and o-DMABN determined by picosecond laser SPC measurements are single-exponential, even in a strongly polar solvent such as acetonitrile (Fig. 3 ).

(a)
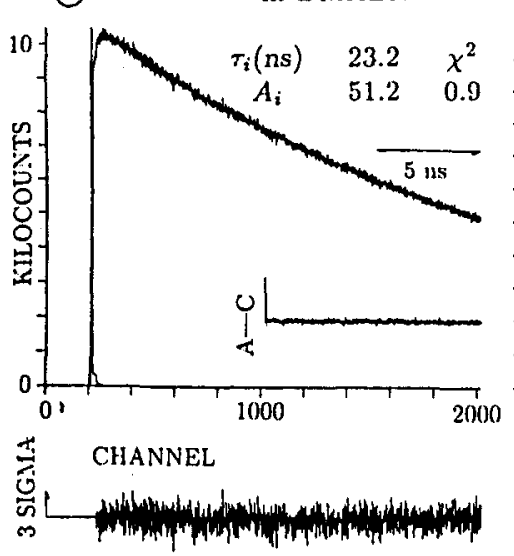

(b)

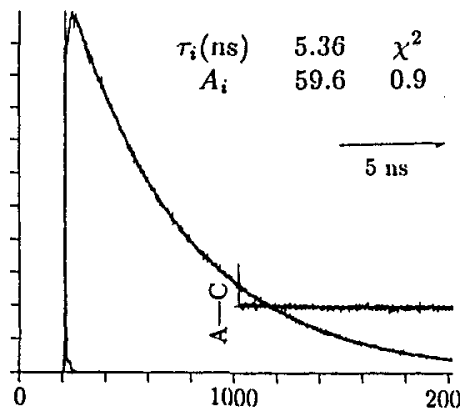

CHANNEL (c)

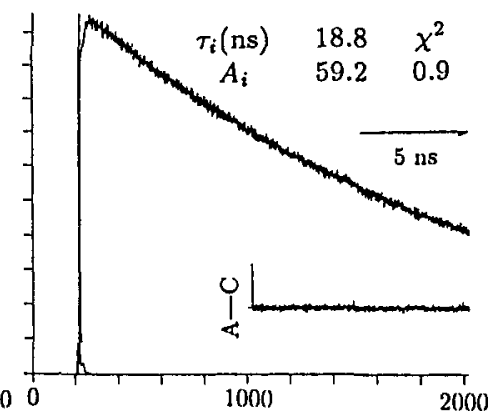

CHANNEL
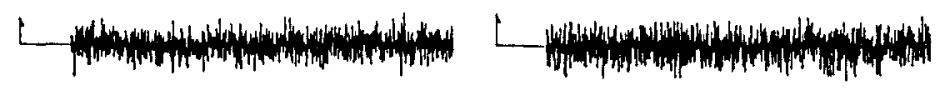

Fig. 3. Fluorescence (LE) decay curves of (a) m-DMABN, (b) o-DMABN and (c) 3,5-DCDMA in acetonitrile at $25^{\circ} \mathrm{C}$.

In the context of the TICT model and its 'principle of minimum overlap' (ref. 5), which postulate that in the TICT-state the dimethylamino and benzonitrile moieties are uncoupled by a rotation of $90^{\circ}$ around the amino-phenyl bond, the complete absence of dual fluorescence with m-DMABN and o-DMABN is unexpected. The uncoupled moieties in the three dimethylaminobenzonitriles would then have similar electron donor and acceptor properties, which should, at least in polar solvents such as acetonitrile or methanol, bring the CT state energetically below the LE state. This conclusion a fortiori holds for 3,5-dicyano- $\mathrm{N}, \mathrm{N}$-dimethylaniline (3,5-DCDMA), in which the 1,3-dicyanobenzene moiety is a much better electron acceptor $\left(E_{1 / 2}=-1.75 \mathrm{~V}\right.$ vs SCE) than benzonitrile $\left(E_{1 / 2}=-2.35 \mathrm{~V}\right.$ vs SCE), ref. 6. For 3,5-DCDMA, dual fluorescence likewise could not be observed, see Fig. 3c, which again is attributed to the relatively large energy gap between $S_{1}$ and $S_{2}$.

\section{3,5-Dimethyl-4-aminobenzonitriles}

The fluorescence spectra of 3,5-dimethyl-4-(methylamino)benzonitrile (MHD) in three solvents of different polarity, n-pentane, diethylether and acetonitrile, are shown in Fig. 4. Although in n-pentane no indication for the presence of dual fluorescence is found, the spectrum of MHD in diethylether 
double-exponential, growing-in from zero at the long-wavelength side of the spectrum with a rise time of 22 ps at $-50^{\circ} \mathrm{C}$ (ref. 8).

\section{Absorption spectra MMD, MHD and HHD}

The absorption spectrum of HHD in n-pentane (Fig. 6a), with a relatively weak structured band next to a stronger structureless band, is similar to the spectrum of ABN in n-pentane (Fig. 2a), showing that the energy gap between the excited states $S_{1}$ and $S_{2}(C T)$ is larger for HHD than for MHD and MMD. The spectrum of MHD in n-pentane, however, is comparable to that of DMABN and not to that of MABN, see Figs. 2 and 6.
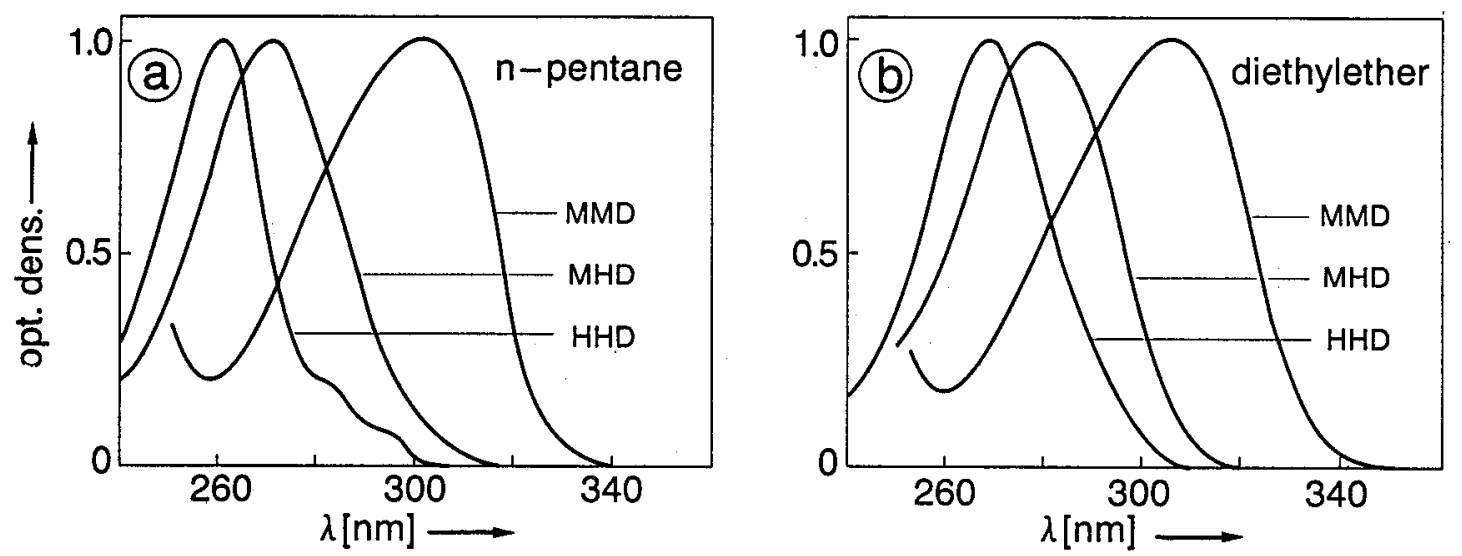

Fig. 6. Absorption spectra of MMD, MHD and HHD in (a) n-pentane and (b) diethylether at $25^{\circ} \mathrm{C}$.

The same conclusion as to the similarity of the absorption spectra and hence of the energy gap between $S_{1}$ and $S_{2}(C T)$ of MHD and DMABN can also be drawn for the solvent diethylether (Fig. $2 \mathrm{~b}$ and $6 \mathrm{~b})$. It can therefore be concluded that the energy gap between $S_{1}$ and $S_{2}(C T)$ for MHD is smaller than that for HHD, becoming similar to the situation prevailing with DMABN as described earlier. The appearance of dual fluorescence with MHD in diethylether and solvents of larger polarity, hence supports our conclusion that the proximity between the energy levels $S_{1}$ and $S_{2}(C T)$ is a requirement for dual fluorescence in the aminobenzonitriles.

In accordance with this interpretation, it has been shown by linear dichroism spectroscopy (ref. 9) that the order between the energies of $S_{1}$ and $S_{2}(C T)$ is reversed in MMD, $S(C T)$ now becoming the lowest excited state. In the case of MMD, the energy gap between $S_{2}$ and the lowest state $S_{1}(C T)$ therefore increases with solvent polarity, in contrast to what is the case with DMABN where the gap between $S_{1}$ and $S_{2}(C T)$ decreases under these conditions. This explains our observation that the LE/CT fluorescence intensity ratio of MMD decreases with solvent polarity, as shown in Fig. 5. This different ordering of the energy levels also explains the observation that in a supersonic jet an excited state reaction occurs with MMD (ref. 10a), but is absent with DMABN (ref. 10b) for which the energy gap between the $S_{1}$ and $S_{2}(C T)$ states in the gas phase apparently is too large for effective vibronic coupling that could lead to a new ICT state, as described in the following section.

\section{Solvent-induced pseudo-Jahn-Teller coupling}

The conclusion that a proximity between the energies of the $S_{1}$ and $S_{2}(C T)$ levels in the aminobenzonitriles is a requirement for the occurrence of ICT, leads to the hypothesis that the dual fluorescence in cyano-substituted anilines such as DMABN is brought about by a solvent-induced pseudo-Jahn-Teller coupling (ref. 11) between $S_{1}$ and $S_{2}(C T)$. The zero order states $\mid n\left(q, Q_{0}\right)>$ for $S_{1}$ and $\mid m\left(q, Q_{0}\right)>$ for $S_{2}(C T)$, where $q$ is the electron coordinate at the equilibrium nuclear configuration $Q_{0}$, could then be coupled by the out-of-plane inversion mode $Q_{p}$ of the amino nitrogen, with the potential energy 
<smiles>Cc1cc(C#N)cc(C)c1C</smiles><smiles>Cc1cc(C#N)cc(C)c1N</smiles><smiles>Cc1cc(C)cc(C#N)c1</smiles>

clearly contains a contribution from a red-shifted CT-emission. As described above, such an emission could not be detected with MABN, likewise containing a $-\mathrm{NH}\left(\mathrm{CH}_{3}\right)$ group.

The CT/LE fluorescence quantum yield ratio $\Phi^{\prime} / \Phi$ further increases with solvent polarity, see the spectrum in acetonitrile in Fig. 4c. $\Phi^{\prime} / \Phi$ also increases with decreasing temperature, reaching, as an example, a maximum with a value of 0.95 at around $-60^{\circ} \mathrm{C}$ in diethylether (ref. 8).
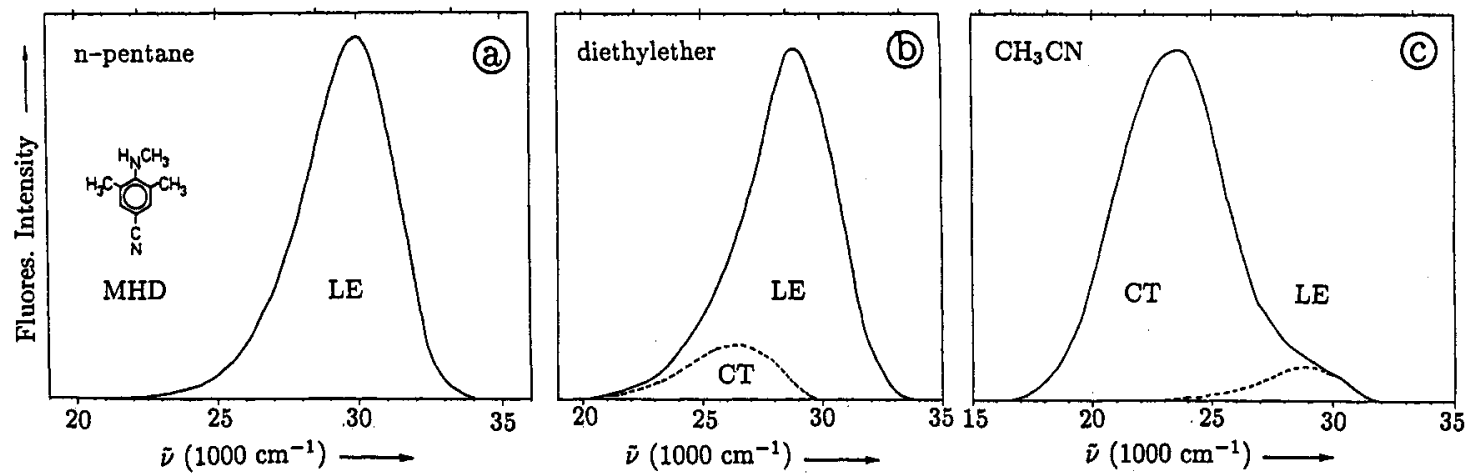

Fig. 4. Fluorescence spectra of MHD in (a) n-pentane, (b) diethylether and (c) acetonitrile at $25^{\circ} \mathrm{C}$.

With 3,5-dimethyl-4-aminobenzonitrile (HHD) dual fluorescence is not observed, even not in strongly polar solvents. The spectrum of 3,5-dimethyl-4-(dimethylamino)benzonitrile (MMD), on the contrary, consists in all solvents investigated of a $\mathrm{CT}$ emission band as the major component. In n-pentane at $25^{\circ} \mathrm{C}$ the fluorescence band has a double maximum and is considerably broader (FWHM $=6200$ $\left.\mathrm{cm}^{-1}\right)$ than in solvents of larger effective polarity such as toluene $\left(5000 \mathrm{~cm}^{-1}\right)$ or acetonitrile $(4900$ $\left.\mathrm{cm}^{-1}\right)$, see Fig. 5. This means that the spectrum of MMD consists of two emission bands, i.e., MMD in n-pentane is dual fluorescent, indicating the presence of an initially excited LE state that gives rise to a CT state in the same manner as has been reported for DMABN in toluene and other solvents (ref. 3,7). In support of this conclusion, the fluorescence decays of MMD in n-pentane are interaction matrix element $U_{m n}^{(p)}$, eq 1 .

$$
U_{m n}^{(p)}=\left\langle m\left(q, Q_{0}\right)\left|\delta U\left(q, Q_{p}\right) / \delta Q_{p}\right| n\left(q, Q_{0}\right)>\right.
$$

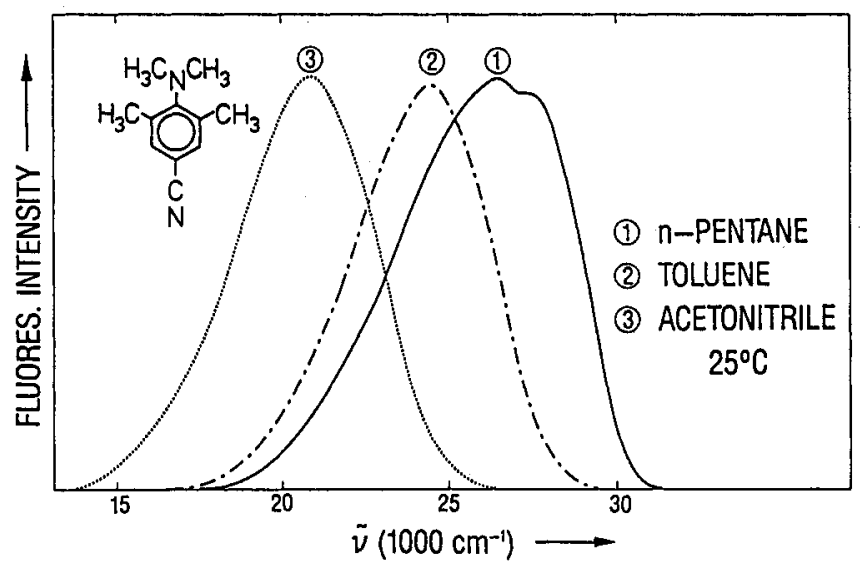

Fig. 5. Fluorescence spectra of MMD in (1) n-pentane, (2) toluene and (3) acetonitrile at $25^{\circ} \mathrm{C}$. 
This vibronic coupling is similar in principle to the 'proximity effect' (ref. 12) studied extensively for the ${ }^{3} n \pi^{*} /{ }^{3} \pi \pi^{*}$ vibronic interaction in nitrogen-heterocyclic and aromatic carbonyl compounds.

\section{Absence of dual fluorescence in rigid planar aminobenzonitriles}

The importance of the N-inversion as a promoting mode for ICT in the aminobenzonitriles could explain the well-known (ref. 2) observation that dual fluorescence does not occur with 1-methyl-5-cyanoindoline (NMCI) and 1-methyl-6-cyano-1,2,3,4-tetraquinoline (NMCQ), in which the 5-membered or 6-membered ring connecting the amino nitrogen with the phenyl group hinders the nitrogen inversion.
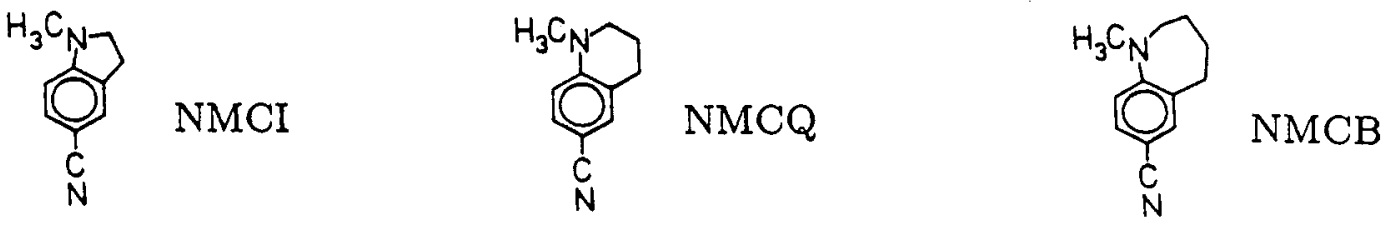

When, however, the aliphatic ring becomes more flexible, as in the benzazepine compound NMCB with a 7-membered ring, ICT and dual fluorescence again starts to appear (ref. 13).

\section{CONCLUSION}

Requirements for the occurrence of dual fluorescence and ICT in aminobenzonitriles are: a sufficiently small energy gap between two interacting states $\left(S_{1}\right.$ and $S_{2}(C T)$ in DMABN) leading to solventinduced pseudo-Jahn-Teller coupling with an amino-nitrogen inversion as the promoting mode.

\section{REFERENCES}

1. (a) E. Lippert, W. Lüder and H. Boos, in: Advances in Molecular Spectroscopy, ed. A. Mangini, pp. 443-457, Pergamon Press, Oxford (1962). (b) E. Lippert, W. Lüder, F. Moll, H. Nagele, H. Boos, H. Prigge and I. Siebold-Blankenstein, Angew. Chem. 73, 695-706 (1961).

2. (a) K. Rotkiewicz, K. H. Grellmann and Z. R. Grabowski, Chem. Phys. Lett. 19, 315-318 (1973). (b) Z. R. Grabowski, K. Rotkiewicz, A. Siemiarczuk, D. J. Cowley and W. Baumann, Nouv. J. Chim. 3, 443-454 (1979).

3. (a) U. Leinhos, W. Kühnle and K. A. Zachariasse, J. Phys. Chem. 95, 2013-2021 (1991). (b) W. Schuddeboom, S. A. Jonker, J. M. Warman, U. Leinhos, W. Kühnle and K. A. Zachariasse, submitted.

4. C. J. Seliskar, O. S. Khalil and S. P. McGlynn, in: Excited States, ed. E. C. Lim, vol.1, pp. 231-294, Academic Press, New York (1974).

5. Z. R. Grabowski and J. Dobkowski, Pure Appl. Chem., 55, 245-251 (1983).

6. P. H. Rieger, I. Bernal, W. H. Reinmuth and G. K. Fraenkel, J. Am. Chem. Soc., 85, 683-693 (1963).

7. J. Hicks, M. Vandersall, Z. Babarogic and K. B. Eisenthal, Chem. Phys. Lett. 116, 18-24 (1985).

8. A. Hebecker, W. Kühnle and K. A. Zachariasse, unpublished results.

9. J. Herbich, K. Rotkiewicz, J. Waluk, B. Andresen and E. W. Thulstrup, Chem. Phys. 138, 105-113 (1989).

10. (a) T. Kobayashi, M. Futakami and O. Kajimoto, Chem. Phys. Lett. 141, 450-454 (1987). (b) R. Howell, H. Petek, D. Phillips and K. Yoshihara, Chem. Phys. Lett. 183, 249-253 (1991).

11. (a) R. Hochstrasser and C. A. Marzzacco, in: Molecular Luminescence, ed. E. C. Lim, pp. 631-655, Benjamin, New York (1969). (b) A. J. Duben, L. Goodman and M. Koyanagi, in: Excited States, ed. E. C. Lim, vol.1, pp. 295-329, Academic Press, New York (1974).

12. E. C. Lim, J. Phys. Chem. 90, 6770-6777 (1991).

13. E. A. Chandross and F. R. Wight, Book of Abstracts. VIth IUPAC Symposium on Photochemistry, Aix-en-Provence 1976, pp. 53-55. 


\section{ERRATA}

1. Pure \& Appl. Chem., Vol. 65, No. 3, pp. 515-520, 1993

The corrected Figure 2, given hereunder, may please be pasted over the incorrect one on page 518 .
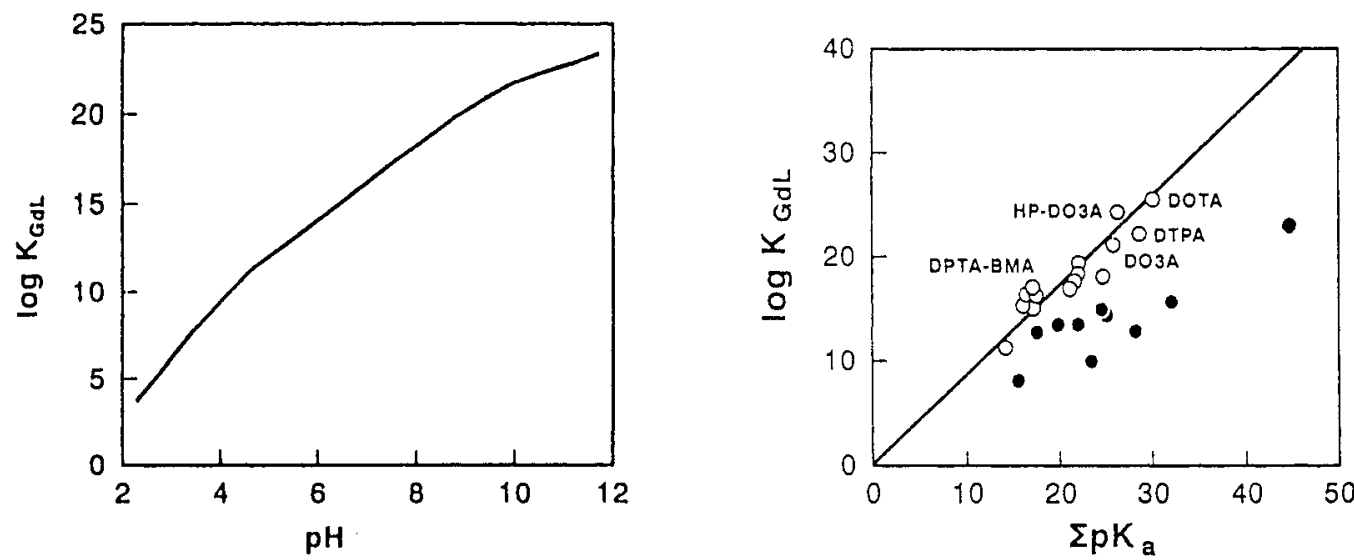

Figure 2 (a) Corrclation of $\log \mathrm{K}_{\mathrm{GdL}}$ with $\Sigma \mathrm{pKa}$ of the neutral ligand. (b) Plot of $\log \mathrm{K}_{\mathrm{GdL}}$ vs. pH for HP-DO3A.

2. Pure \& Appl. Chem., Vol. 65, No. 8, pp. 1745-1750, 1993

The corrected pages 1748 and 1749, which follow, may please be pasted over the incorrect ones. 
<smiles>Cc1cc(C#N)cc(C)c1C</smiles><smiles>Cc1cc(C#N)cc(C)c1N</smiles><smiles>Cc1cc(C)cc(C#N)c1</smiles>

clearly contains a contribution from a red-shifted CT-emission. As described above, such an emission could not be detected with MABN, likewise containing a $-\mathrm{NH}\left(\mathrm{CH}_{3}\right)$ group.

The CT/LE fluorescence quantum yield ratio $\Phi^{\prime} / \Phi$ further increases with solvent polarity, see the spectrum in acetonitrile in Fig. 4c. $\Phi^{\prime} / \Phi$ also increases with decreasing temperature, reaching, as an example, a maximum with a value of 0.95 at around $-60^{\circ} \mathrm{C}$ in diethylether (ref. 8).
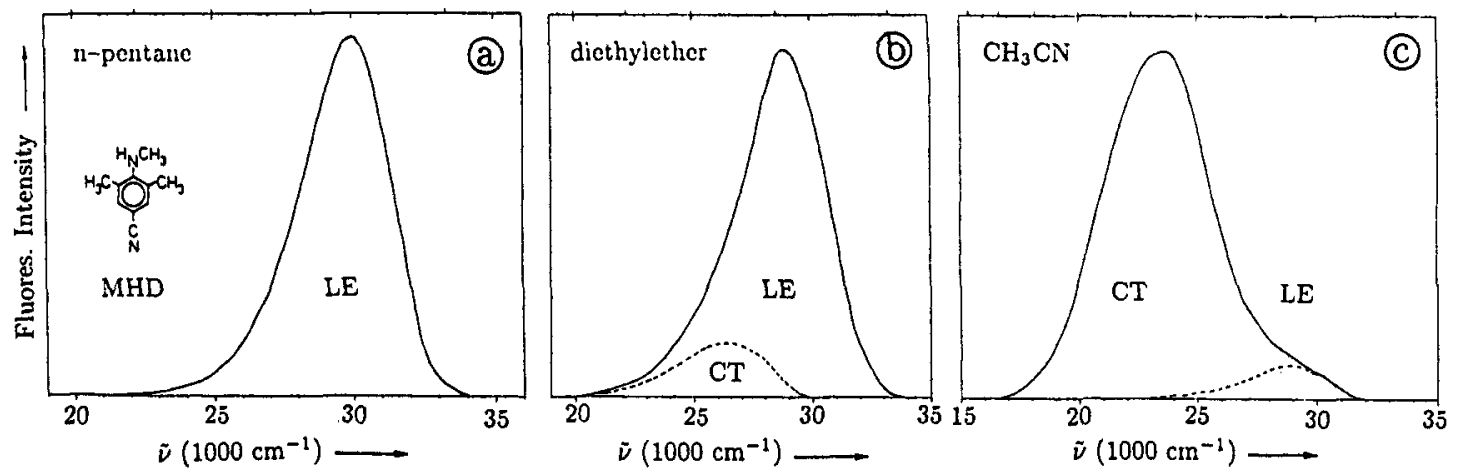

Fig. 4. Fluorescence spectra of MHD in (a) n-pentane, (b) diethylether and (c) acetonitrile at $25^{\circ} \mathrm{C}$.

With 3,5-dimethyl-4-aminobenzonitrile (HHD) dual fluorescence is not observed, even not in strongly polar solvents. The spectrum of 3,5-dimethyl-4-(dimethylamino)benzonitrile (MMD), on the contrary, consists in all solvents investigated of a $\mathrm{CT}$ emission band as the major component. In n-pentane at $25^{\circ} \mathrm{C}$ the fluorescence band has a double maximum and is considerably broader (FWHM $=6200$ $\left.\mathrm{cm}^{-1}\right)$ than in solvents of larger effective polarity such as toluene $\left(5000 \mathrm{~cm}^{-1}\right)$ or acetonitrile $(4900$ $\mathrm{cm}^{-1}$ ), see Fig. 5. This means that the spectrum of MMD consists of two emission bands, i.e., MMD in n-pentane is dual fluorescent, indicating the presence of anl initially excited LE state that gives rise to a CT state in the same manner as has been reported for DMABN in toluene and other solvents (ref. 3,7). In support of this conclusion, the fluorescence decays of MMD in n-pentane are double-exponential, growing-in from zero at the long-wavelength side of the spectrum with a rise time of 22 ps at $-50^{\circ} \mathrm{C}$ (ref. 8 ).

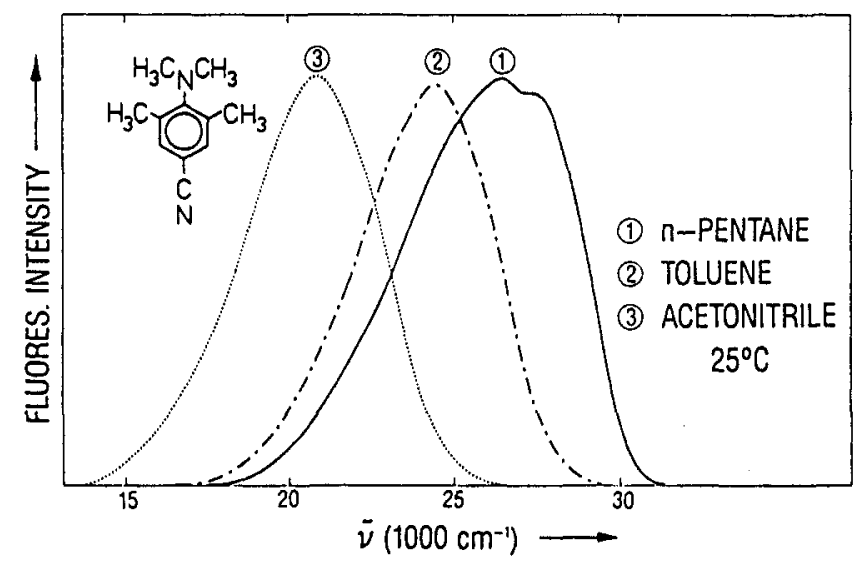

Fig. 5. Fluorescence spectra of MMD in (1) n-pentane,

(2) toluene and (3) acetonitrile at $25^{\circ} \mathrm{C}$. 


\section{Absorption spectra MMD, MHD and HHD}

The absorption spectrum of HHD in n-pentane (Fig. 6a), with a relatively weak structured band next to a stronger structureless band, is similar to the spectrum of $A B N$ in n-pentane (Fig. 2a), showing that the energy gap between the excited states $S_{1}$ and $S_{2}(C T)$ is larger for HHD than for MHD and MMD. The spectrum of MHD in n-pentane, however, is comparable to that of DMABN and not to that of MABN, see Figs. 2 and 6.
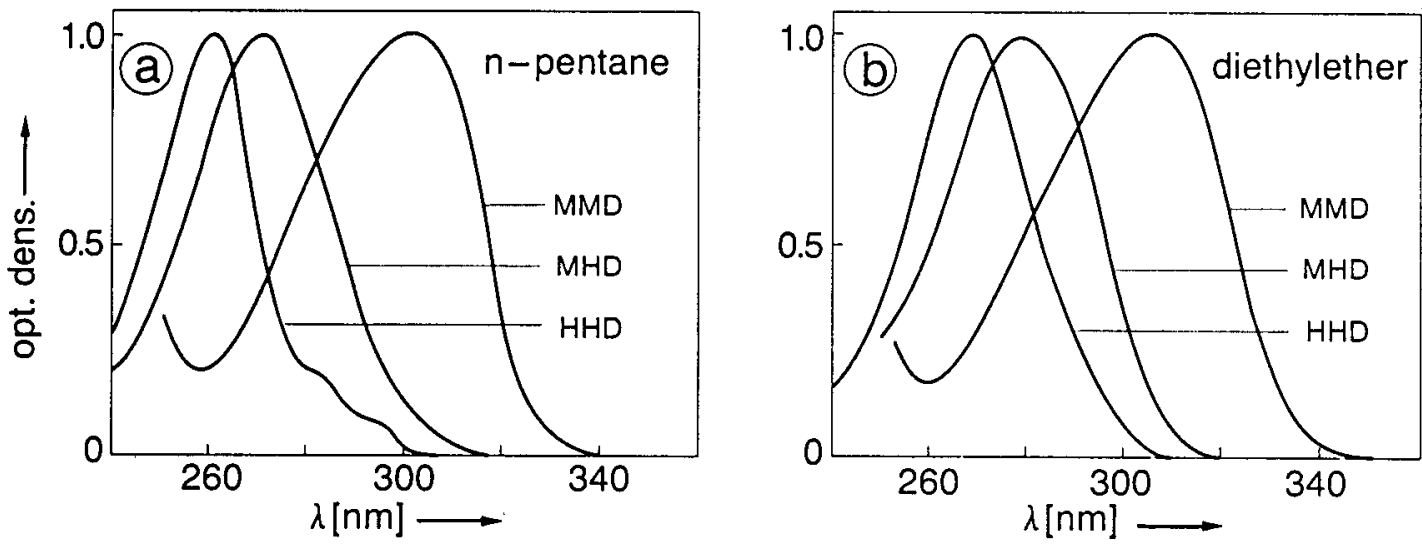

Fig. 6. Absorption spectra of MMD, MHD and HHD in (a) n-pentane and (b) diethylether at $25^{\circ} \mathrm{C}$.

The same conclusion as to the similarity of the absorption spectra and hence of the energy gap between $S_{1}$ and $S_{2}(C T)$ of MHD and DMABN can also be drawn for the solvent diethylether (Fig. $2 \mathrm{~b}$ and $6 \mathrm{~b})$. It can therefore be concluded that the energy gap between $S_{1}$ and $S_{2}(C T)$ for MHD is smaller than that for HHD, becoming similar to the situation prevailing with DMABN as described earlier. The appearance of dual fluorescence with MHD in diethylether and solvents of larger polarity, hence supports our conclusion that the proximity between the energy levels $S_{1}$ and $S_{2}(C T)$ is a requirement for dual fluorescence in the aminobenzonitriles.

In accordance with this interpretation, it has been shown by linear dichroism spectroscopy (ref. 9) that the order between the energies of $S_{1}$ and $S_{2}(C T)$ is reversed in MMD, $S(C T)$ now becoming the lowest excited state. In the case of MMD, the energy gap between $S_{2}$ and the lowest state $S_{1}(C T)$ therefore increases with solvent polarity, in contrast to what is the case with DMABN where the gap between $S_{1}$ and $S_{2}(C T)$ decreases under these conditions. This explains our observation that the LE/CT fluorescence intensity ratio of MMD decreases with solvent polarity, as shown in Fig. 5 . This different ordering of the energy levels also explains the observation that in a supersonic jet an excited state reaction occurs with MMD (ref. 10a), but is absent with DMABN (ref. 10b) for which the energy gap between the $S_{1}$ and $S_{2}(C T)$ states in the gas phase apparently is too large for effective vibronic coupling that could lead to a new ICT state, as described in the following section.

\section{Solvent-induced pseudo-Jahn-Teller coupling}

The conclusion that a proximity between the energies of the $S_{1}$ and $S_{2}(C T)$ levels in the aminobenzonitriles is a requirement for the occurrence of ICT, leads to the hypothesis that the dual fluorescence in cyano-substituted anilines such as DMABN is brought about by a solvent-induced pseudo-Jahn-Teller coupling (ref. 11) between $S_{1}$ and $S_{2}(C T)$. The zero order states $\mid n\left(q, Q_{0}\right)>$ for $S_{1}$ and $\mid m\left(q, Q_{0}\right)>$ for $S_{2}(C T)$, where q is the electron coordinate at the equilibrium nuclear configuration $Q_{o}$, could then be coupled by the out-of-plane inversion mode $Q_{p}$ of the amino nitrogen, with the potential energy interaction matrix element $U_{m n}^{(p)}$, eq 1 .

$$
U_{m n}^{(p)}=\left\langle m\left(q, Q_{0}\right)\left|\delta U\left(q, Q_{p}\right) / \delta Q_{p}\right| n\left(q, Q_{0}\right)>\right.
$$

